Mitteilungen der Österreichischen Geographischen Gesellschaft, 161. Jg., S. 357-378

(Annals of the Austrian Geographical Society, Vol. 161, pp. 357-378)

Wien (Vienna) 2019, https://doi.org/10.1553/moegg161s357

\title{
175 JAhre VerLag Ed. Hölzel \\ Innovation aus Tradition. Eine Geschichte des Verlags \\ Ed. Hölzel, 1844-2019
}

Barbara N. WIESINGER, Wien*

mit 11 Abb. im Text

\section{INHALT}

1 Der Buchhändler und Verleger Eduard Hölzel 357

2 Die Entwicklung zum Schulbuchverlag mit Schwerpunkt Geographie und Kartographie (1850-1918/19) 358

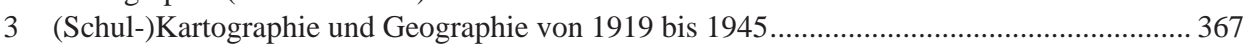

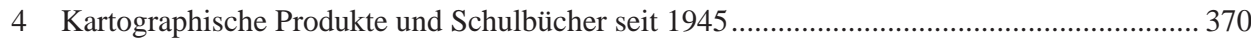

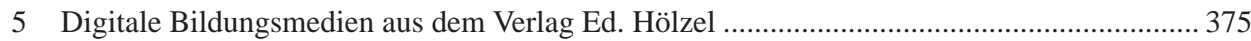

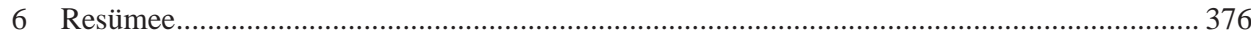

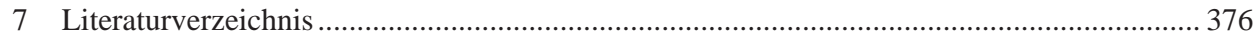

\section{Der Buchhändler und Verleger Eduard Hölzel}

Eduard HöLzel wurde am 8. Oktober 1817 in Prag [Praha] geboren. Sein Vater Thomas HöLzeL (1788-1846/47) war ein kunst- und naturbegeisterter Eisenwarenhändler. Seine Mutter Margarethe, geb. HerLth (1795-1823), verstarb jung. Eduard Hölzel wuchs deshalb unter der Obhut seiner Stiefmutter Thekla HöLzel, geb. Srboví (Lebensdaten unbekannt), auf. Thekla HöLzel, die aus einer reichen Braumeisterfamilie stammte, vermittelte ihren Kindern ihre vielseitigen kulturellen Interessen. Nach dem Abschluss des Gymnasiums absolvierte Eduard HöLzel eine Buchhändlerlehre in der Prager Verlagsbuchhandlung Borrosch \& André (gegründet 1824). Als Geselle arbeitete er in Leipzig, Mainz, Augsburg, Würzburg, Wien und Brünn [Brno], bis er 1844 in Olmütz [Olomouc] seine erste eigene Buch-, Kunst- und Musikalienhandlung gründete. Diese wurde schnell zu einem Mittelpunkt des kulturellen Lebens in der mährischen Festungs- und Universitätsstadt. Im Februar 1848 heiratete Eduard Hölzel Hedwig NiemanN (1827-1881), die Tochter eines Brünner Offiziers mit bürgerlicher Verwandtschaft in Olmütz. Von Eduard und Hedwig HöLzels neun Kindern erreichten nur vier das Erwachsenenalter: Hugo Josef, geb. 1852; Adolf Richard, geb. 1853; Emma Maria Antonia, geb. 1855; Hedwiga Amalia, geb. 1857. Hedwig HöLzel teilte die Interessen ihres Mannes und unterstützte seine beruflichen und intellektuellen Ambitionen u. a. als Gastgeberin von Literatur- und Diskussionsabenden (KuBIČKoví 2017a, S. 350-354).

\footnotetext{
* Dr. Barbara N. Wiesinger, Verlag Ed. Hölzel GmbH Nfg KG, Jochen-Rindt-Straße 9, 1230 Wien; E-Mail: wiesinger@hoelzel.at
} 
Eduard HöLzel publizierte seit 1845 Belletristik, Fachliteratur und Schulbücher in tschechischer und deutscher Sprache, Lithographien (Einzelblätter und Alben) von regionalen Sehenswürdigkeiten sowie geographische Karten. Von 1848 bis 1858 verlegte er außerdem die liberale Zeitung Die neue Zeit. Zwischen 1849 und 1871 entstanden weitere Buchhandlungen in Mähren und schließlich in Wien. 1861 erwarb HöLzel die Lithographische Kunstanstalt K. Horegschi (tschech. Karel HoŘEGŠí) in der Luisengasse 5 (heute Mommsengasse) im 4. Wiener Gemeindebezirk und gründete hier sein Geographisches Institut. Seither befindet sich der Firmensitz des Verlags Ed. Hölzel in Wien (KuBIČKOv'́ 2017b, S. 18, 24; 2019, S. 93-94).

Im Jahr 1871 zog die Familie Hölzel dauerhaft nach Wien, wo sich der Verleger Eduard HöLzeL als Standesvertreter - er war 1859 Mitbegründer und 1868 bis 1875 Vorsitzender des Vereins der österreichisch-ungarischen Buchhändler - sowie Förderer von Wissenschaft, Bildung und Kunst einen Namen machte. Insbesondere bemühte er sich um die Geographie und Kartographie. Bereits 1857 war er zum Mitglied der 1856 gegründeten Kaiserlich-Königlichen Geographischen Gesellschaft in Wien (heutiger Name: Österreichische Geographische Gesellschaft, ÖGG) ernannt worden. HöLzel war mit führenden Erdwissenschaftlern seiner Zeit bekannt, etwa mit dem Geologen Eduard Suess (1831-1914), dem Geologen Franz von Hauer (1822-1899), dem Geographen Friedrich Simony (1813-1896), dem Geologen Ferdinand von Hochstetter (1829-1884), dem Geographen Karl KoRISTKA (tschech. Karel Kořistka, 1825-1906) und dem Geographielehrer und Kartographen Blasius Kozenn (slowen. Blaž Kocen, 1821-1871).

Aus diesen Kontakten gingen zahlreiche erfolgreiche Verlagsprodukte hervor. Das prominenteste Beispiel dafür ist der „Kozenn-Atlas“ (erste Auflage 1861), der - immer wieder neu konzipiert und aktualisiert - noch heute an Österreichs Schulen verwendet wird (BIRSAK 2011). Eduard HöLzeLs berühmte Serie großformatiger Farblithographien „Geographische Charakterbilder“ (erschienen ab 1881), die im Austausch mit der k. k. Geographischen Gesellschaft konzipiert worden war, prägte das Weltbild des gebildeten Publikums des 19. Jahrhunderts (JovanOvic-KruSPEL 2019). Für seine Verdienste als Verleger erhielt Eduard HöLzel zahlreiche nationale und internationale Auszeichnungen. Er starb am 21. Dezember 1885 in Salzburg und ist am Wiener Zentralfriedhof begraben (KuBIČKoví 2019, S. 93; BIRSAK und WiESINGER 2019, S. 9, 29).

Nach Eduard HöLzels Tod übernahm sein Sohn Hugo Hölzel (1852-1895) gemeinsam mit seinem Schwager Emil Kosmack (1840-1893) den Verlag nebst Druckerei und Buchhandlungen. Die letzte Besitzerin aus der Gründerfamilie war Hugo HöLzels Witwe Louise Hölzel (gestorben 1954), die das Unternehmen 1899 mehrheitlich an den tschechischen Papierfabrikanten Theodor Eichmann verkaufte. Dessen Erben verkauften ihre Anteile 1935 an das Badener Druck- und Verlagsunternehmen Rudolf M. ROHRER. 1941 wurde das Fürstenhaus LiECHTENSTEIN Mehrheitseigentümer des Verlags Ed. Hölzel. Eine Minderheit hielt Dr. Hugo EcKelT (1894-1976), der den Verlag als Geschäftsführer von 1926 bis zu seinem Tod wesentlich prägte. 1978 wurde der Verlag an die Mayr-Melnhof'sche Industriegruppe verkauft und 1990 von der österreichischen $P \& V$ Holding $A G$ übernommen, in deren Besitz er sich bis heute befindet (BIRSAK und WIESINGER 2019).

\section{Die Entwicklung zum Schulbuchverlag mit Schwerpunkt Geographie und Kartographie (1850-1918/19)}

Als vielseitig interessierter und aufgeklärt-liberaler Unternehmer erkannte Eduard HöLzEL die Bedürfnisse seiner potenziellen Kundschaft, entwickelte passende Produkte und platzierte sie mit wachsendem Erfolg zuerst auf dem regionalen Markt, dann in der gesamten Habsburgermonarchie und schließlich sogar weit darüber hinaus. 
Als Olmützer Buchhändler und Verleger verkaufte und publizierte er tschechische und deutschsprachige Werke. Damit bediente er das Bildungs- und Informationsbedürfnis nicht nur des arrivierten deutschsprachigen Bürgertums in Mähren, sondern auch einer wachsenden bildungsinteressierten und national gesinnten tschechischen bürgerlichen Schicht (НrocH 1996, S. 379, 384, 386). Als der Verlag zunehmend überregional agierte, wurde Sprachenvielfalt, insbesondere für den Schulgebrauch, ein wichtiger Baustein für den Erfolg von Ed. Hölzels Verlagstätigkeit: So listete ein Katalog von 1906 Schulatlanten in sieben Sprachen und geographische Wandkarten in zehn Sprachen und zwei Schriften auf. Lehrbücher erschienen damals in sieben Sprachen der Habsburgermonarchie sowie auf Englisch und Französisch (Verlag Ed. Hölzel 1906).

Einen Namen machte sich der Verlag ab den 1850er Jahren aber vor allem mit künstlerisch und drucktechnisch hochwertigen Farblithographien. Das damals hochmoderne Verfahren der Lithographie (auch: Steindruck, Ölfarbendruck) hatte Eduard HöLzel über seinen Vater kennengelernt, der mit dessen Erfinder Alois SENEFELDER (1771-1834) befreundet gewesen war. Eduard HöLzeL erkannte früh das kommerzielle Potenzial, das die Lithographie in Hinblick auf die insbesondere bildungsbürgerliche „Seh(n)sucht des 19. Jahrhunderts nach Bildern“ (JovanOvic-KruSPEL 2019, S. 100) eröffnete. Um 1860 verlegte er erstmals getönte Ansichten von regionalen Landschaften und Bauwerken. ${ }^{1)}$ Außerdem veröffentlichte Eduard HöLzEL bereits seit den 1850er Jahren farbig illustrierte naturwissenschaftliche Fachbücher. ${ }^{2)}$

Der Verlag Ed. Hölzel reproduzierte und vermarktete auch Werke alter Meister und zeitgenössischer Künstler. Diese Lithographien wurden auf Leinwand aufgezogen, gefirnisst und gerahmt verkauft und schmückten bürgerliche Interieurs nicht nur in Mitteleuropa, sondern sogar in den USA, bis die Nachfrage nach solchen Reproduktionen in den 1870er Jahren zurückging (KLEE 2019, S. 96; JovanOVIC-KRUSPEL 2019, S. 100).

Bereits in den 1870er Jahren erkannte Eduard Hölzel das „Defizit von Sichtbarkeit“ (AltHAUS 2018, zit. nach JovANOVIC-KRUSPEL 2019, S. 99), das die bildungsaffine Gesellschaft des 19. Jahrhunderts angesichts des rapiden wissenschaftlichen Fortschritts prägte. Visualisierungen waren damals gefragt, um die neuen Erkenntnisse vor allem der Naturwissenschaften breit rezipieren, diskutieren und weiterentwickeln zu können. Insbesondere farbige Illustrationen verbesserten die Verständlichkeit und Attraktivität und damit die Breitenwirksamkeit von gelehrten Abhandlungen für Schule, Universität und Arbeitswelt und leisteten damit einen wesentlichen Beitrag zu Bildungsexpansion und Wissenschaftspopularisierung.

Als Antwort auf dieses gesellschaftliche Bedürfnis konzipierte Eduard HöLzEL im Austausch mit Künstlern und Wissenschaftlern kommerziell erfolgreiche und von der Kritik gewürdigte Wandbilderserien mit religiösen, (kunst)historischen und geographischen Sujets: die „Bilder zur Geschichte“ (ab 1873) nach Vorlagen des Künstlers Joseph LangL (1843-1916), die „Biblischen Bilder“ (ab 1878) nach Originalen des Malers Ernst PessLeR (1838-1900) und die in Zusammenarbeit mit Friedrich Simony (1813-1896) und Ferdinand von HochstetTer (1829-1884) konzipierten und von der k. k. Geographischen Gesellschaft geförderten „Geographischen Charakterbilder“ (ab 1881) nach

1) Zum Beispiel das „Malerisch-historische Album von Mähren und Schlesien“ (Olmütz 1857-1860) und das „Malerisch-historische Album vom Königreich Böhmen“ (Olmütz 1857-1860).

2) Ferdinand FisCALI „Deutschlands Forstcultur-Pflanzen“ (Olmütz 1856) mit 18 Farbtafeln; Friedrich MARQUART „Die eßbaren und schädlichen Schwämme“ (Olmütz 1856) mit vier Farbtafeln von Anton MüLLER; Ferdinand FISCALI „Die schädlichen Forstinsekten“ (Wien/Olmütz 1857) mit zwei Farbtafeln ebenfalls von Anton MüLler; Karl B. Heller „Naturgeschichtlicher Schul-Atlas für die k. k. Gymnasien und Realschulen“ (Wien 1857) mit zahlreichen Farbtafeln eines unbekannten Künstlers sowie Theodor KотschY „Die Eichen Europa‘s und des Orient‘s“ (Wien/Olmütz 1858-1861) mit Farbtafeln von Johann Oberer und Josef SebotHA. Die Lithographien für diese Werke wurden bei A. Hartinger bzw. bei K. Horegschi in Wien produziert (KuBIČKOVA 2017c, S. 67-83). 
Vorlagen verschiedener Künstler wie Karl HASCH (1834-1897), Rudolf ReSCHREITER (1868-1939), Friedrich BECK (1873-1921), Ludwig Hans FisCHER (1848-1915) u. a. Überaus erfolgreich waren auch die für den Einsatz in der Grundschule gedachten „Wandbilder für den Anschauungs- und Sprachunterricht“ (ab 1884), die zumindest teilweise nach Vorlagen der mährischen Künstlerinnen Marie GöRLICH (1851-1896) und Sophie GöRLICH (1855-1893) entstanden sind (BIRSAK und WIESINGER 2019, S. 25, 29-34).

Das Besondere an Ed. Hölzels Wandbildern war, dass sie das kulturelle Erbe Europas („Bilder zur Geschichte“), Erkenntnisse aus der neuen Leitwissenschaft Geographie („Geographische Charakterbilder“, Abb. 1) sowie ein für die breiten Massen gedachtes Weltwissen (“Anschauungsbilder“, Abb. 2) in Lithographien gossen, die ästhetisch dem populären Zeitgeschmack entsprachen und technisch in bester Qualität ausgeführt waren. Da sie massenhaft reproduziert wurden, waren sie für Bildungsinstitutionen ebenso wie für Privathaushalte erschwinglich und entfalteten so eine immense Breitenwirkung. Günstige Handausgaben ermöglichten eine noch größere Verbreitung dieser lehrreichen Lithographien. ${ }^{3)}$

Ab den 1860er Jahren entwickelte sich die (Schul-)Geographie und insbesondere die (Schul-) Kartographie zur wichtigsten Kernkompetenz des Verlags Ed. Hölzel. 1858 hatte Eduard HöLzeL den aus dem heutigen Slowenien stammenden Geographen Blasius KozenN (1821-1871) kennengelernt, der damals am Olmützer Gymnasium unterrichtete. Kozenn entstammte einer Bauernfamilie, konnte durch Förderung aber Gymnasium und Universität besuchen. In Klagenfurt absolvierte

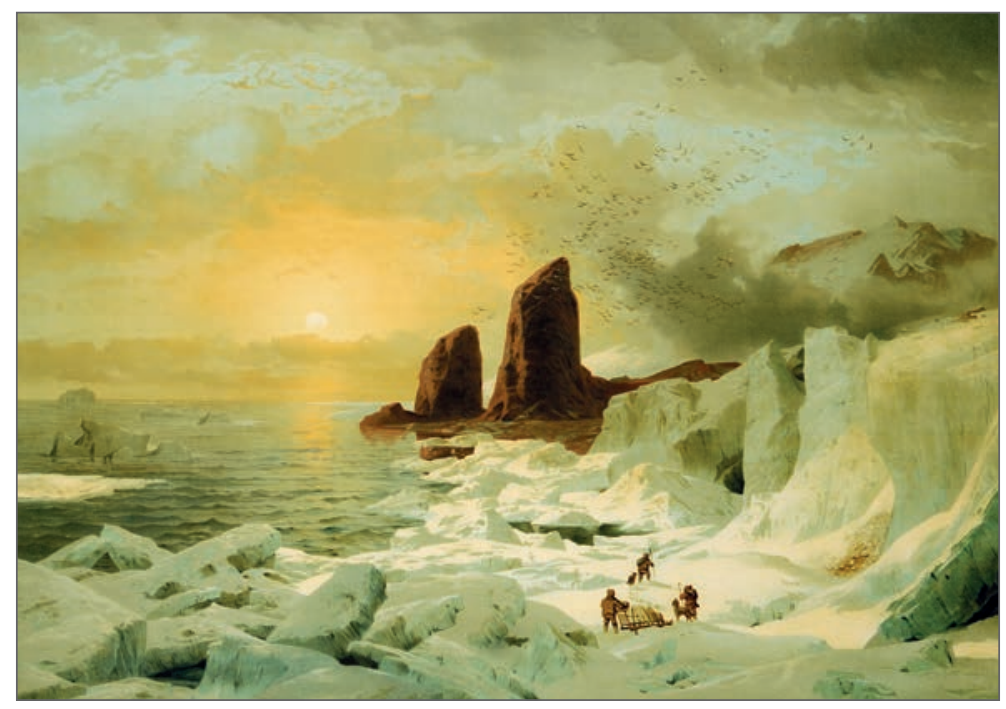

Quelle: Archiv Verlag Ed. Hölzel, Foto: A. SchUMACHER

Abb. 1: „Säulenkap auf Kronprinz-Rudolphs-Land (Franz-Josephs-Land)“ [Rudolf-Insel, FranzJosef-Land] nach einer Vorlage von Karl Hasch aus der Serie „Geographische Charakterbilder“, o. J.

\footnotetext{
3) „Hölzel’s Geographische Charakterbilder. Kleine Handausgabe“ mit Begleittexten von Friedrich UmLAUFT und Vinzenz vON HAARDT, Wien 1887; „Hölzel's Bilderbuch“ mit acht Anschauungsbildern und Begleittext von Eduard JORDAN, Wien 1887.
} 


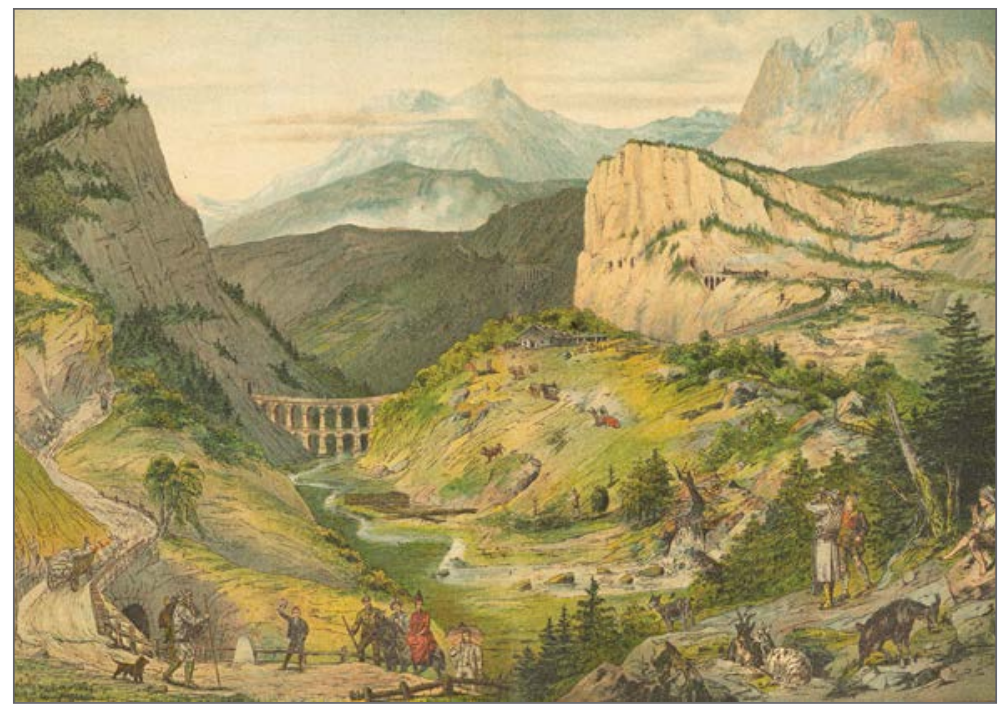

Quelle: Archiv Verlag Ed. Hölzel, Foto: A. Schumacher

Abb. 1: „Das Gebirge“ aus der Serie „Wandbilder für den Anschauungs- und Sprachunterricht“, o. A., o. J.

Kozenn das Priesterseminar, erhielt die Priesterweihe und war einige Jahre als Pfarrer tätig. Sein Lehramtsstudium absolvierte er in Wien (BrateC Mrvar et al. 2011, S. 13-20).

Blasius Kozenn konzipierte und realisierte für Ed. Hölzel den „Geographischen Schul-Atlas für die Gymnasien, Real- und Handelsschulen der österreichischen Monarchie“, der 1861 erstmals erschien. Die 31 mehrfarbigen und teilweise aufklappbaren Kartenblätter der ersten Auflage wurden bereits in der hauseigenen Druckerei in Wien unter Leitung des Lithographen Friedrich KöKE hergestellt. Dabei waren die bisher im Verlag gesammelten Erfahrungen mit der Farblithographie nützlich, denn der Druck vielfarbiger geographischer Karten war äußerst komplex. Auf eine Doppelseite mit Erdansichten folgten im ersten „Kozenn-Atlas“ jeweils eine physische und eine politische Übersichtskarte der Kontinente (Abb. 3), dann ausgewählte Regionen und Staaten im Detail sowie abschließend die Kronländer der Monarchie. Ergänzt wurde das Kartenwerk durch Erläuterungen zu Kartenprojektionen, Terrainlehre und geographischen Begriffen bzw. Namen. Bereits 1862 erschien eine gekürzte Ausgabe des „Kozenn-Atlas“ mit nur acht Kartenblättern, die für Grundschulen intendiert war.

In den Folgejahren wurde der „Kozenn-Atlas“ nicht nur wiederholt aktualisiert und erweitert, sondern auch mit Erläuterungen in tschechischer, ungarischer und polnischer Sprache versehen. Dies trug sicherlich dazu bei, dass sich der „Kozenn-Atlas“ schließlich gegenüber den Produkten des deutschen Mitbewerbs, insbesondere dem „Stieler-Atlas“ (vgl. Siтte W. 2001, S. 411), auf dem österreichisch-ungarischen Markt durchsetzen konnte (BIRSAK und WIESINGER 2019, S. 19). Gegenüber seinem Atlaswerk gerieten Blasius KozenNs Lehrbücher ${ }^{4)}$ in den Hintergrund.

4) B. KozENNs „Erdbeschreibung für Volksschulen“ erschien bei Ed. Hölzel auf Deutsch, Tschechisch und Slowenisch und erlebte ab 1858 mindestens 14 Auflagen, der „Leitfaden der Geographie für Schulen im Kaiserthum Österreich“ (erstmals erschienen bei Ed. Hölzel 1868, später unter dem Titel „Leitfaden der Geographie für den Unterricht“) erlebte sogar 21 Auflagen (Bratec Mrvar et al. 2011, S. 28; SitTe Ch. 1987, S. 142). 


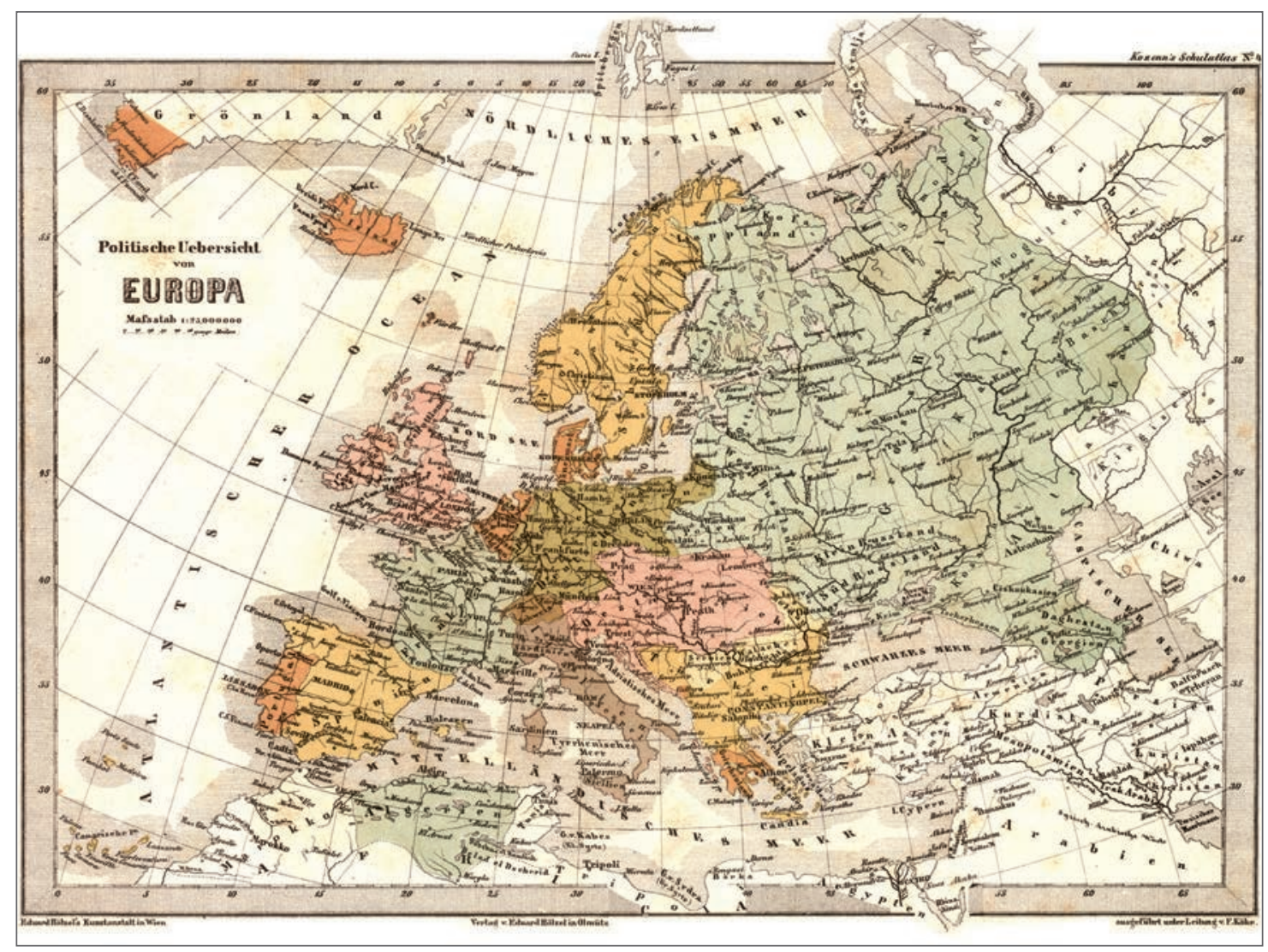

Die „Ausbrüche“ (Spitzbergen und Nowaja Semlja im Norden, Halbinsel Sinai im Süden) aus dem Satzspiegel ergeben sich durch Buchformat, Kartenmaßstab und physische Gestalt des abzubildenden Erdteils oder Landes. Sie wurden ein typisches Merkmal der Hölzel-Kartographie.

Quelle: Archiv Verlag Ed. Hölzel

Abb. 3: „Politische Übersicht von Europa, 1 : 25000 000“. Nr. 4 aus dem „Kozenn-Atlas“, 1861

Nach dem frühen Tod Blasius Kozenns 1871 gewann Eduard HöLzel 1877 den Wiener Geographen, Lehrer und Volksbildner Friedrich UmLauft (1844-1923) als Bearbeiter für den „Kozenn“. UMLAUFT hatte bei Friedrich Simony, Universitätsprofessor für Geographie an der Universität Wien und Hölzel-Autor, studiert und leitete 1899-1919 die Wiener Urania. Außerdem verfasste er zahlreiche Reiseberichte, Fachartikel und Lehrbücher für Geographie. Seit der 23. Auflage von 1878 orientierte der „Kozenn-Atlas“ Karten von Regionen außerhalb Europas am Nullmeridian von Greenwich. 1880 übernahm der Kartograph Vinzenz HaARDT von HARTENTHURn (1843-1914) die Bearbeitung des „Kozenn-Atlas“. Er leitete von 1887 bis 1896 die Kartographie im Verlag Ed. Hölzel und wechselte dann an das Wiener Militärgeographische Institut. Er betreute nicht nur den „Kozenn“, sondern erarbeitete auch einen „Volksschulatlas“ für Ed. Hölzel, der in verschiedenen regionalen Ausgaben erhältlich war (DAMMERER 2007, S. 39ff; BIRSAK und Wiesinger 2019, S. 23, 27). Um 1900 hatte sich der „Kozenn-Atlas“ schließlich an den Schulen der Habsburgermonarchie etabliert (SITTE W. 2001, S. 412). 


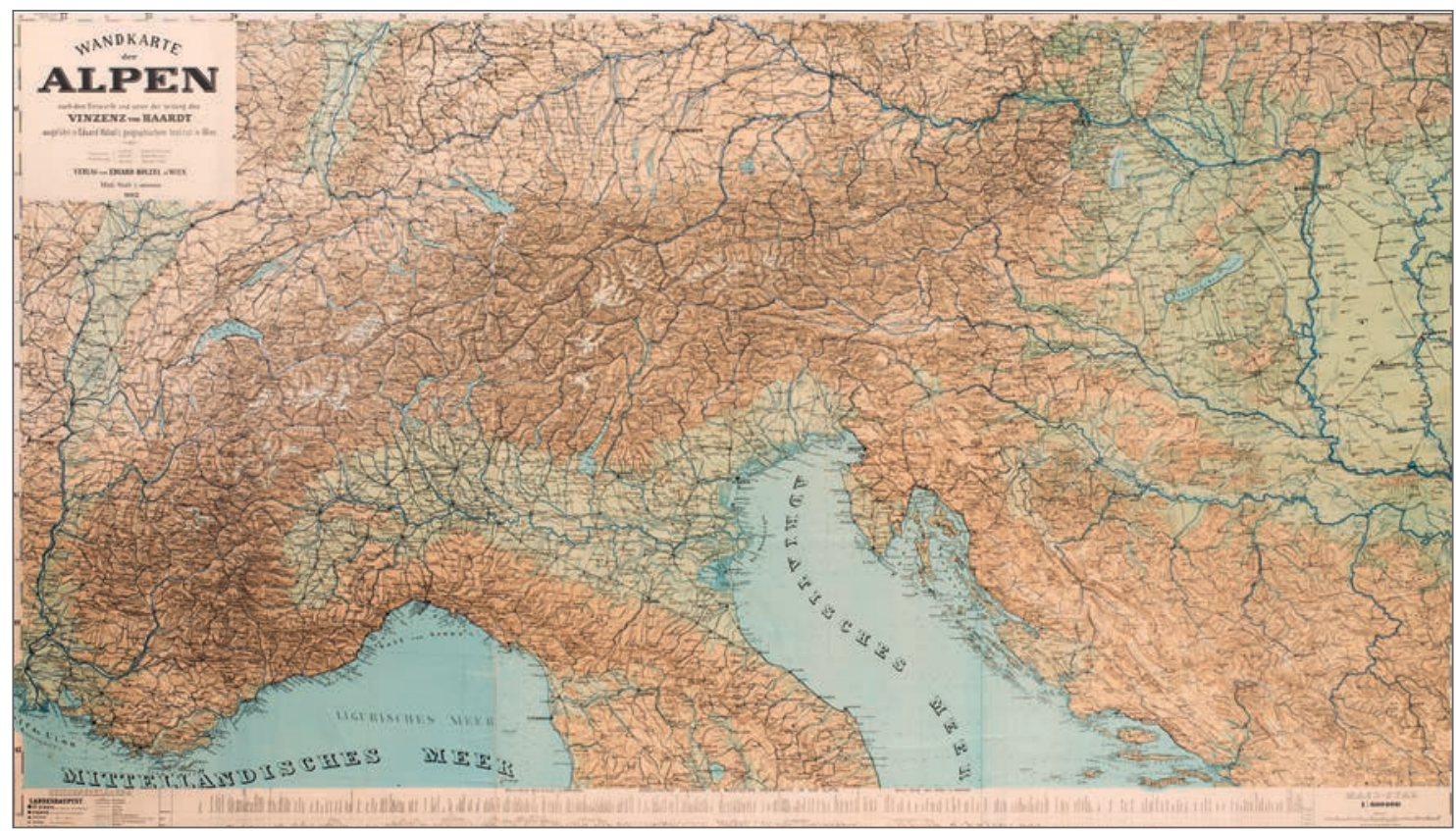

Quelle: $\quad$ Archiv Verlag Ed. Hölzel, Foto: U. HANTSCH

Abb. 4: „Wandkarte der Alpen 1 : 600 000“ (Ausschnitt) von Vinzenz von HAARDT, 1882

Bereits in Zusammenarbeit mit Blasius KozenN waren bei Ed. Hölzel als Ergänzung zu den Schulatlanten geographische Wandkarten für den Schulunterricht entstanden, zum Beispiel eine zwischen 1861 und 1867 zu datierende Wandkarte Europas in tschechischer Sprache sowie eine nach 1867 zu datierende Wandkarte Europas in ungarischer Sprache. 1882 veröffentlichte der Verlag dann die wegweisende „Wandkarte der Alpen“ im Maßstab 1 : 600000 von Vinzenz von HAARDT (Abb. 4). Diese zeichnete sich außer durch das große Format und den dadurch ermöglichten großen Maßstab durch eine besonders plastische Geländedarstellung und eine harmonische Abstimmung von Färbigkeit und Schraffen aus (DAmmerer 2007, S. 42 und S. 150ff). 1906 führte der Verlag bereits zahlreiche Wandkarten der Erde, der Kontinente, der Kronländer und Regionen der Habsburgermonarchie sowie diverse Spezialkarten für den Religions- und Geschichtsunterricht in zehn verschiedenen Sprachen, über 50 allein auf Deutsch (Verlag Ed. Hölzel 1906, S. 8-17).

Als nach dem Tod des Verlagsgründers 1885 dessen Sohn Hugo HöLzel und sein Schwager Emil Kosmack das Unternehmen übernahmen, erweiterte sich das Sortiment um teuer ausgestattete Fachbücher, Reiseberichte und Länderbeschreibungen, die auch schon zahlreiche Fotografien beinhalteten. Zu erwähnen ist zum Beispiel das Werk „Das Dachsteingebiet: ein geographisches Charakterbild“ (1889) des bereits erwähnten Alpenforschers und Universitätsprofessors für Geographie Friedrich Simony) (BirsaK und Wiesinger 2019, S. 31, 35; VuKović 2019).

5) Die aufwändigste Publikation dieser Periode war das dreiteilige Prachtwerk „Die Bäume und Sträucher des Waldes“ des Forstwissenschaftlers Gustav Hempel (1842-1904) und des Botanikers und Mykologen Karl WiLHELm (1848-1933) mit zahlreichen farbigen Tafeln. 
Damals erschien auch einer der wenigen stärker wissenschaftlich und thematisch statt nur topographisch orientierten Atlanten aus dem Hause Ed. Hölzel: der „Physikalisch-statistische Hand-Atlas von Österreich-Ungarn“ (1887) mit anschaulichen Karten verschiedener Autoren sowohl zur physischen Geographie als auch zur Bevölkerungs- und Wirtschaftsstatistik (Abb. 5). Ab 1887 publizierte Ed. Hölzel auch seinen ersten Geschichte-Atlas: den „Historisch-geographischen Schul-Atlas“ (Abb. 6) von Dr. Wilhelm Schmidt und Friedrich Schubert (erste Gesamtausgabe 1899), der mit Karten bis in das späte 19. Jahrhundert hochaktuell war (BIRSAK und WiESINGER 2019, S. 37).

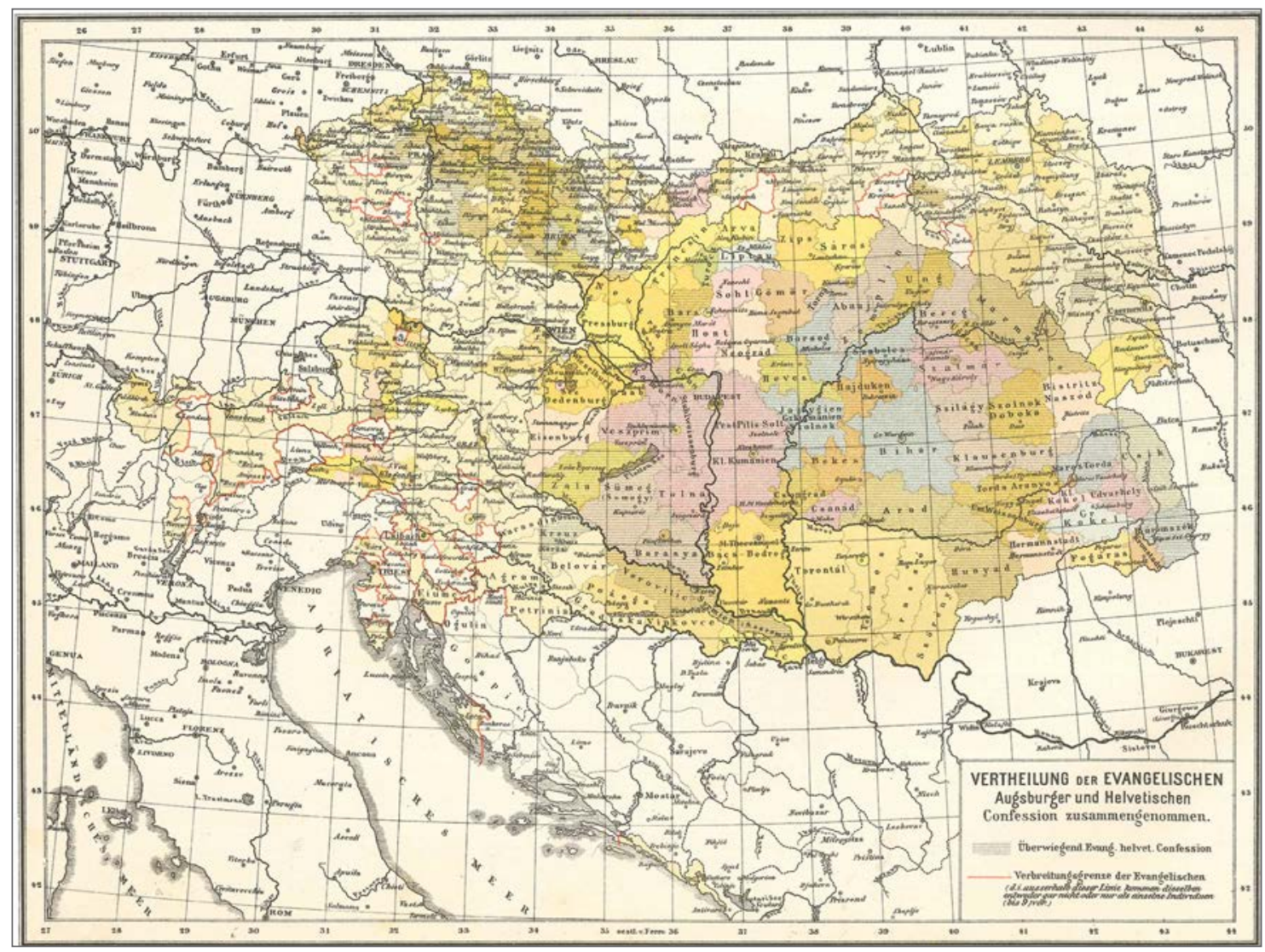

Die Legende zu den vier Karten zur Religionsverteilung (katholische, evangelische und orthodoxe Christen sowie Israeliten) ist außerhalb der Karten gedruckt und hier nicht abgebildet.

Quelle: Archiv Verlag Ed. Hölzel

Abb. 5: „Vertheilung der Evangelischen“ - Karte 16/2 von Dr. Franz Ritter von Le MonNier aus dem „Physikalisch-statistischen Hand-Atlas von Österreich-Ungarn“, 1887

Erst Wilhelm ZwIERZINA, der den Verlag von 1895 bis 1914 leitete, legte den Tätigkeitsschwerpunkt wieder auf Schulbuch und Schulkartographie. Er gewann 1901 den Geographen Dr. Franz HeIDERICH (1863-1926) als Bearbeiter für den „Kozenn-Atlas“. HeIDERICH, der ab 1905 an der Exportakademie [spätere Bezeichnung: Hochschule für Welthandel, heute: Wirtschaftsuniversität 


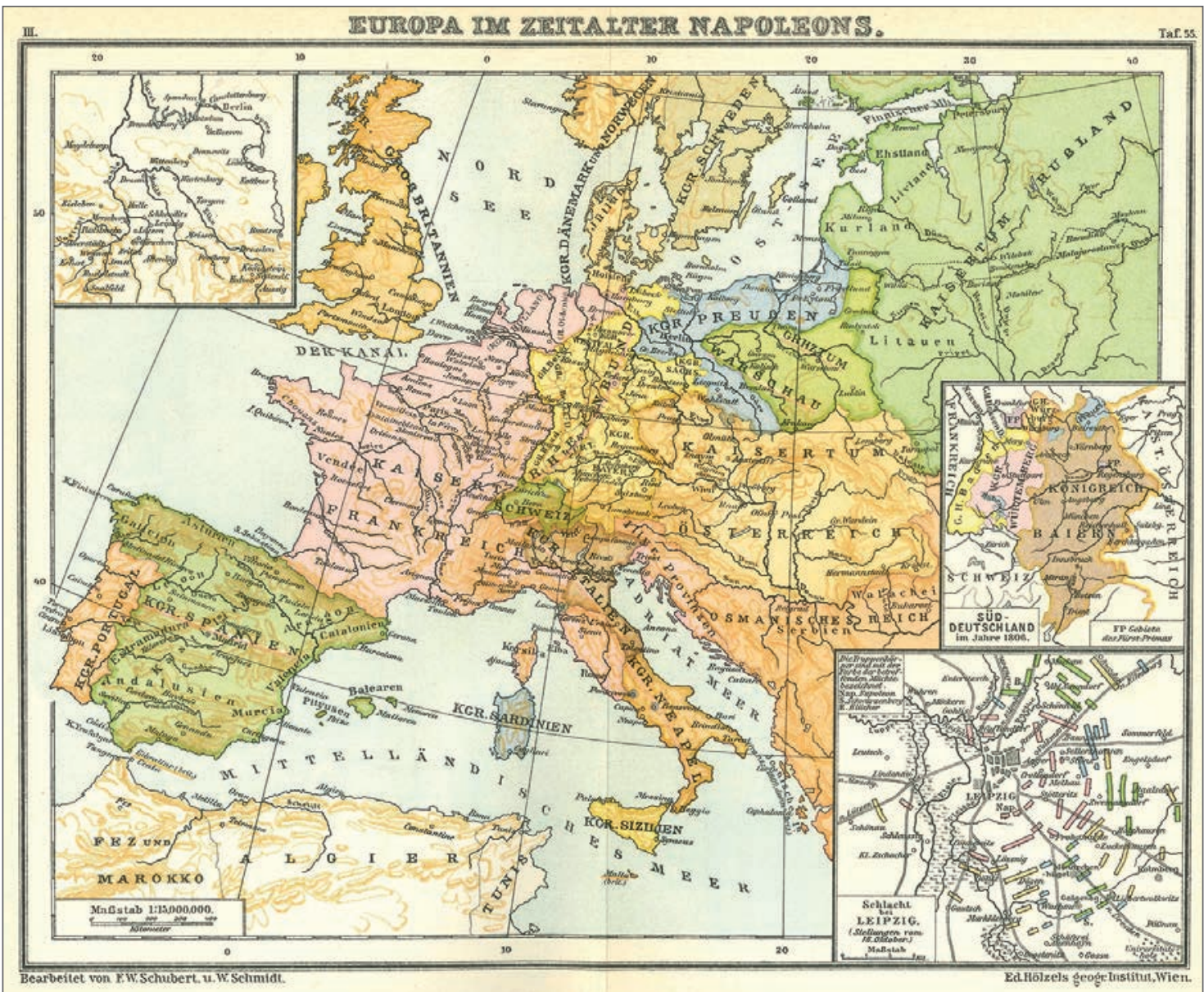

Quelle: Archiv Verlag Ed. Hölzel

Abb. 6: „Europa im Zeitalter Napoleons“, Tafel 55 aus dem „Historisch-geographischen Schulatlas für Mittelschulen“von Wilhelm SchmidT und Friedrich (Franz) SchuBERT, 2. verbesserte und ergänzte Auflage 1927

Wien] Wirtschaftsgeographie lehrte und dort ab 1909 eine Professur bekleidete, brachte erstmals Wirtschaftsthemen in den „Kozenn“ ein. Auch als Autor der Serie „Österreichische Schulgeographie“ in acht Bänden (erschienen ab 1904 und bis in die NS-Zeit in Gebrauch) prägte HeIDERICH die Schulgeographie in Österreich mit.

Die Praxistauglichkeit seiner Serie beruhte auf mehreren Faktoren, die auch heute noch bei der Gestaltung von Schulbüchern eine wichtige Rolle spielen:

- Übersichtliche Gliederung (mehrseitige Kapitel, die wiederum in nummerierte und mit Überschriften ausgezeichnete Paragrafen gegliedert sind),

- Erklärungen von Fachbegriffen und anderen unbekannten Wörtern,

- Hervorhebung wichtiger Begriffe (durch Fettdruck), 
- Veranschaulichung durch zahlreiche Illustrationen im Fließtext sowie durch Bildbeilagen mit kommentierten Schwarz-Weiß-Reproduktionen ausgewählter „Geographischer Charakterbilder“.

Im Vergleich zu modernen Schulbüchern auffällig ist die Prädominanz des erklärenden Fließtextes, während Arbeitsaufträge nur selten vorkommen. Sie beschränken sich zudem auf die Reproduktion der im Fließtext präsentierten Inhalte, wie zum Beispiel „Wiederhole im Zusammenhange die europäischen Staaten und die Flüsse nach den Meeren, in die sie münden“ oder „Wiederhole die asiatischen Flüsse im Zusammenhange“ (HeIDERIch 1910, S. 67 bzw. 71).

Ebenfalls um 1900 eröffnete sich der Verlag mit der Herstellung von Straßenkarten ein neues Tätigkeitsfeld, das das gesamte 20. Jahrhundert hindurch ein wichtiger Baustein des Geschäftserfolgs blieb, bis im beginnenden 21. Jahrhundert digitale Navigationssysteme gedruckte Straßenkarten verdrängten. Ein frühes Beispiel dafür sind die in Zusammenarbeit mit dem k. k. österreichischen Automobilklub herausgegebenen „Automobilkarten für die im Reichsrate vertretenen Königreiche und Länder“ von 1913 (Abb. 7). Während des Ersten Weltkriegs erschienen Hölzels Autokarten im Maßstab 1: 600000 auch gesammelt im „Tauril-Atlas“ (um 1915), einer Kooperation mit einer Firma für Autobedarf.

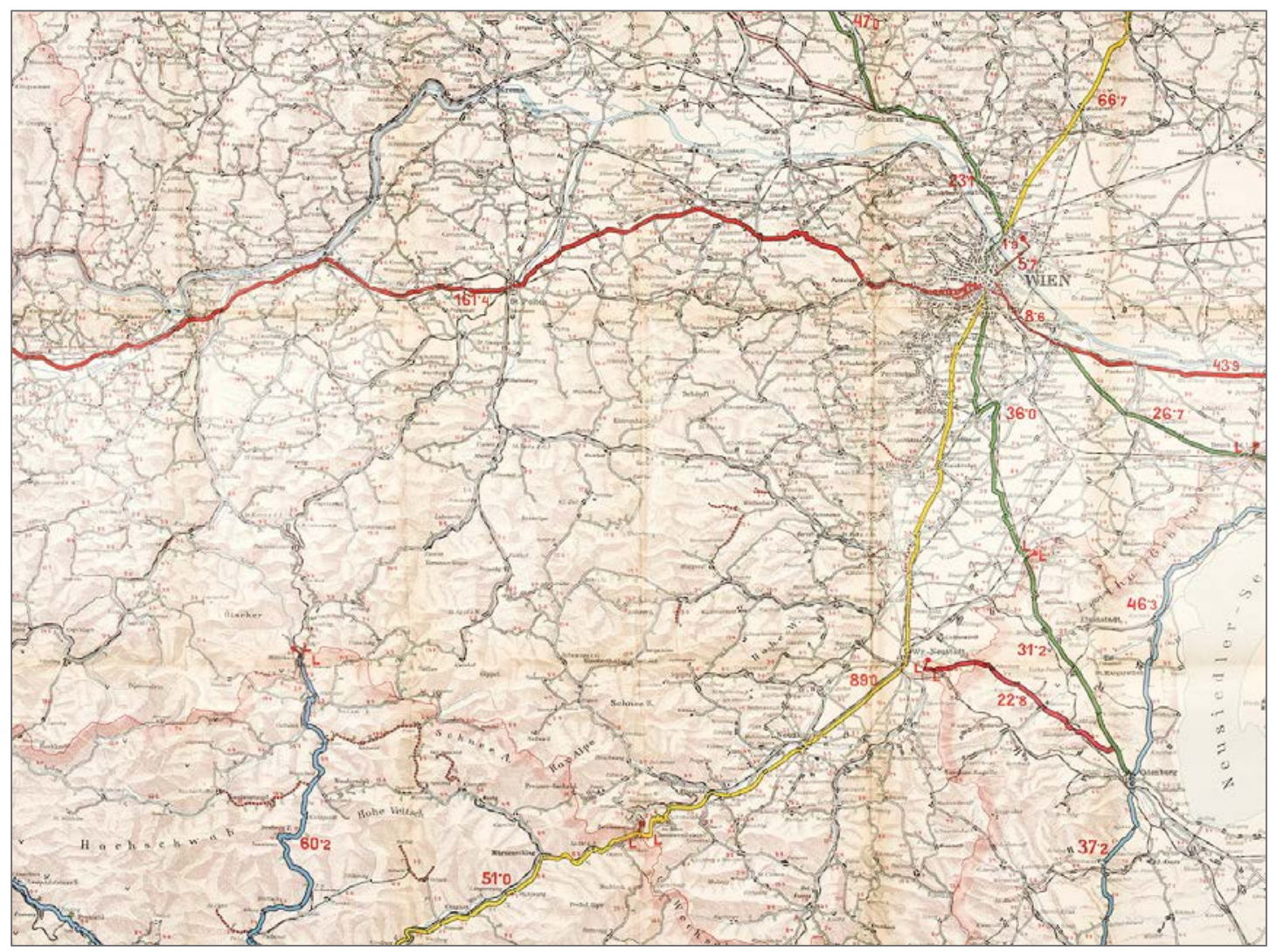

Quelle: Archiv Verlag Ed. Hölzel, Foto: U. Hantsch

Abb. 7: Blatt XIV von „Hölzels Automobilkarten für die im Reichsrate vertretenen Königreiche und Länder“, um 1913 
Der Erste Weltkrieg 1914-1918 war in mehrfacher Hinsicht eine Zäsur in der Verlagsgeschichte: Erstens schied Geschäftsführer Wilhelm ZwIERZINA 1914 nach knapp zwanzigjährigem Wirken aus dem Verlag aus. Zweitens wurden zwischen 1914 und 1916 für alle Kriegsschauplätze Kriegs- und Handkarten erarbeitet, wodurch andere Produkte ins Hintertreffen gerieten. Maßgeblich waren aber schließlich ab 1918/19 die empfindliche Verkleinerung des Marktes durch den Zerfall der Habsburgermonarchie sowie die allgemeine Wirtschaftskrise der Nachkriegszeit. In den 1920er Jahren erfolgte außerdem die Umstellung auf den modernen Offsetdruck. Die teuren neuen Maschinen rentierten sich aber nicht, da die kartographischen Produkte des Verlags nach wie vor im Steindruck hergestellt wurden (BIRSAK und WIESINGER 2019, S. 45f).

\section{3 (Schul-)Kartographie und Geographie von 1919 bis 1945}

Bald gelang es dem Verlag Ed. Hölzel jedoch, sich zusätzlich zum österreichischen Markt neue Absatzgebiete zu erschließen. So wurden im Königreich der Slowenen, Kroaten und Serben (ab 1929: Jugoslawien) von 1919 bis zum Zweiten Weltkrieg kroatische und serbische Lizenzausgaben verschiedener „Kozenn-Atlanten“ vertrieben, und auch in Polen und der Türkei wurde die Hölzel-Kartographie nachgefragt. Üblicherweise wurden die fremdsprachigen Atlanten von einheimischen Experten adaptiert und von nationalen Verlagen vermarktet, während das Kartenmaterial in Österreich erarbeitet wurde - ein Geschäftsmodell, das auch nach 1945 erfolgreich bei Kooperationen mit europäischen und US-amerikanischen Verlagshäusern angewandt wurde.

Die Veränderungen der europäischen Staatenwelt nach 1919 machten damals auch die Erarbeitung neuer politischer Karten notwendig. Um 1921 erschien bei Ed. Hölzel eine Karte der Republik Österreich im Maßstab 1: 1000000 (Abb. 8), etwa 1922 eine neue Handkarte Europas im Maßstab $1: 6000000$ (BIRSAK und Wiesinger 2019, S. 47, 49).

Nachdem der Jurist Dr. Hugo ECKELT 1925/26 die Leitung übernommen hatte, konzentrierte sich der Verlag Ed. Hölzel wieder auf seine Kernkompetenzen (Schul-)Kartographie und Geographie. ECKELT gewann mit dem Landesschulinspektor und Lehrerbildner Dr. Heinrich GüTTENBERGER (1886-1946) und dem Wirtschaftsgeographen Dr. Hermann LeITER (1892-1958), der als Professor an der Wiener Hochschule für Welthandel wirkte, neue Bearbeiter für den „KozennAtlas". 1929 erschien die 50. Auflage, in der erstmals Wirtschaftskarten eine bedeutendere Rolle spielten. GütTENBERGER leitete außerdem die Neuausgabe des „Kozenn-Atlas für Hauptschulen“, die 1930 erschien, und bearbeitete die Schulbuchreihe „Österreichische Schulgeographie“ von Franz HeIDERICH nach den neuen Lehrplänen der Republik. 1929 lagen alle acht Bände aktualisiert vor und blieben bis in den Zweiten Weltkrieg hinein im Schulgebrauch (BIRSAK und Wiesinger 2019, S. 49).

Nach dem „Anschluss“ Österreichs an NS-Deutschland 1938 musste die „Österreichische Schulgeographie“ wie alle anderen Lehrwerke österreichischer Verlage einer NS-Kommission zur Genehmigung vorgelegt werden. Die neuen Machthaber wünschten nämlich ideologiekonforme neue Lehrwerke, waren aber für eine begrenzte „Übergangszeit“ gewillt, die bisherigen österreichischen Schulbücher im Umlauf zu lassen, sofern deren Inhalte ihren Zielen nicht wesentlich widersprachen. Die „Österreichische Schulgeographie“, die aus heutiger Sicht zum Beispiel durchaus als rassistisch zu wertende Textpassagen zur Humangeographie enthält, bestand diese „Prüfung“. Allerdings musste der Titelbestandteil „Österreichische“ gestrichen werden, wie Abbildung 9 zeigt (BIRSAK und WiesINGer 2019, S. 51).

Im Jahr 1935 veröffentlichte der Verlag Ed. Hölzel den „Atlas zur allgemeinen und österreichischen Geschichte“ des Historikers Dr. Wilhelm SchiER, der mit den territorialen Veränderungen durch die Friedensverträge 1919 endete, also hochaktuell war. Der „Schier“ erschien, bearbeitet 


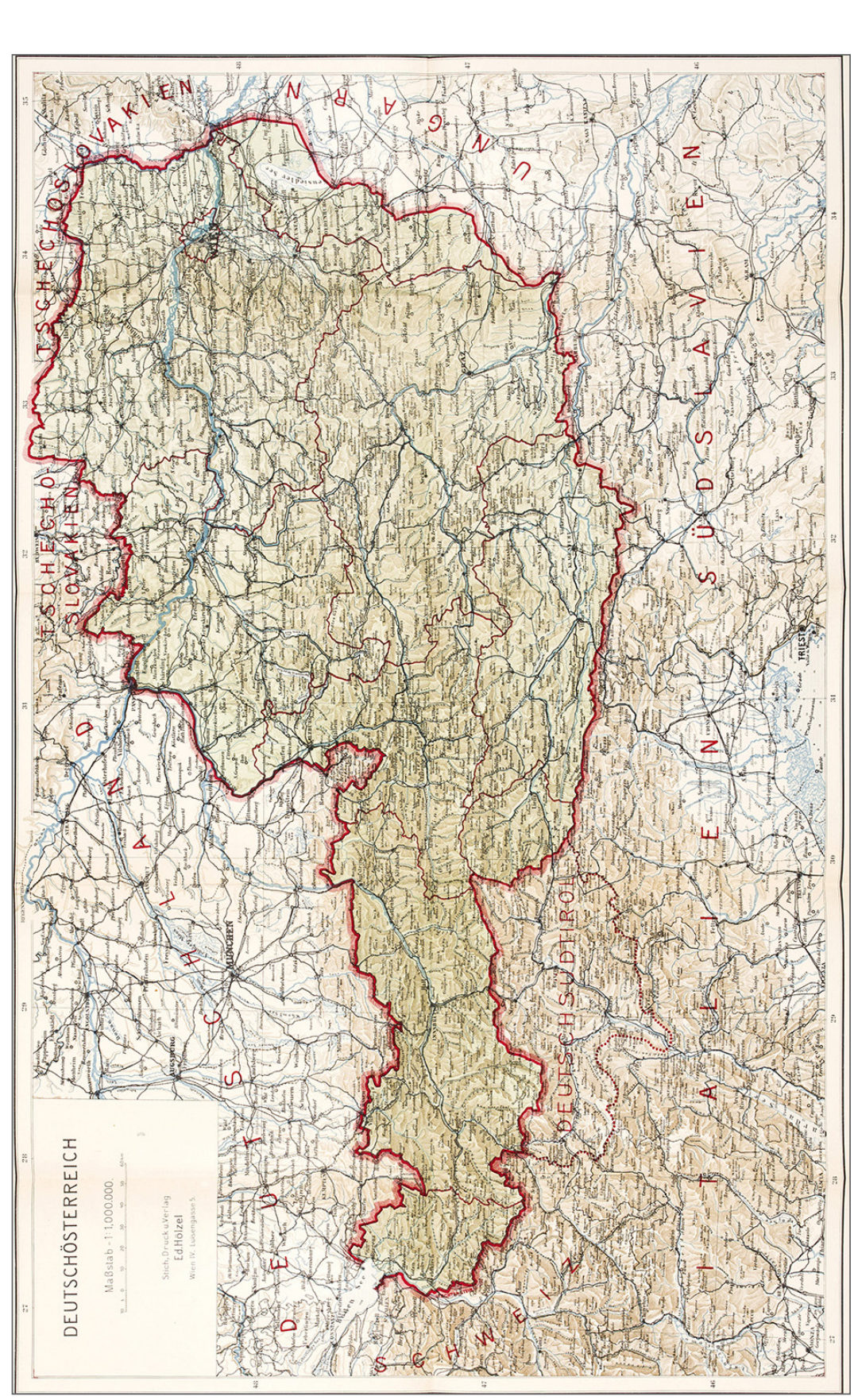

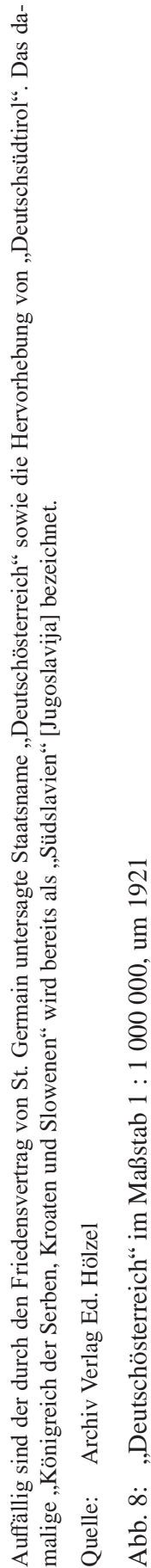




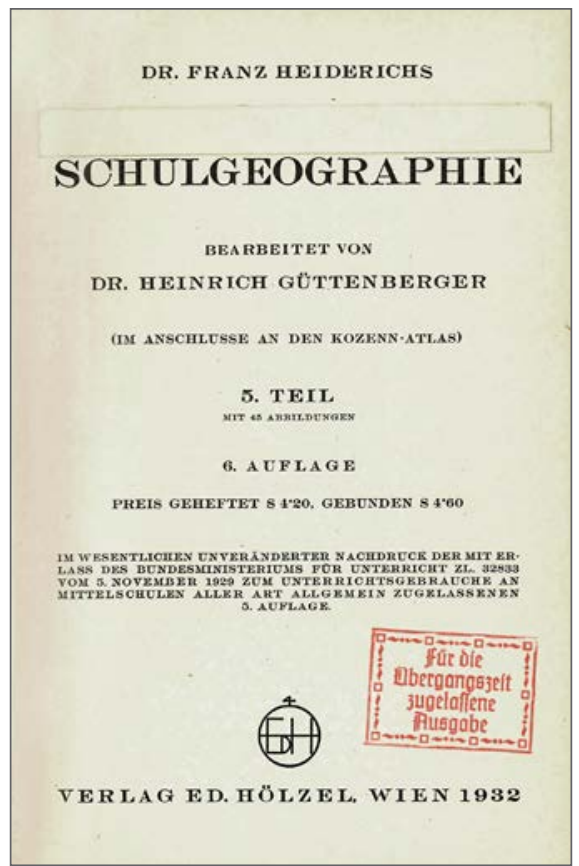

Schmutztitel des Schulbuchs „Österreichische Schulgeographie“, 5. Teil, mit Klebekorrektur über „Österreichische“ und vorgeschriebenem Stempel für den Weitergebrauch während der NS-Zeit. Im Verlagsarchiv sind mehrere Exemplare mit dieser Klebekorrektur erhalten.

Quelle: Archiv Verlag Ed. Hölzel

Abb. 9: Franz HeIderich / Heinrich GütTEnBERGER, „Österreichische Schulgeographie“, 5. Teil, 6. Auflage, Wien 1932

und ergänzt, bis in das Jahr 2005, als spezielle Geschichteatlanten zunehmend von Kombinationsprodukten abgelöst wurden.

1939 verpflichtete der Verlag mit Dr. Hans SLANAR sen. (1890-1955) einen bekannten fortschrittlichen Schulgeographen als leitenden Mitarbeiter der Kartographie. Der Gymnasialdirektor, der in der Ersten Republik für den Wiener Verlag Jugend und Volk erfolgreiche Lehrbücher und Atlanten verfasst hatte, war von der NS-Verwaltung 1938 aus dem Schuldienst entlassen worden. Er betreute den „Kozenn-Atlas“ während der Kriegsjahre und verantwortete auch die erste Nachkriegsauflage (die 75. Auflage) von 1951 (BIRSAK und WiesINGER 2019, S. 59).

Auch der „Kozenn-Atlas“ blieb während des Nationalsozialismus weiterhin an Österreichs Schulen in Gebrauch. Die Karten zu Europa spiegelten die durch den NS-Eroberungskrieg abermals veränderten politischen Verhältnisse wider. In der 59. Auflage von 1941 waren die Österreich-Karten zwar selbst mit „Ostmark“ überschrieben, im Inhaltsverzeichnis stand aber nach wie vor „Österreich“ zu lesen. Eine eigene Karte (S. 118/119) zeigte die der Parteiorganisation der NSDAP entsprungene Aufteilung Österreichs in „Gaue“, wobei die Bundesländer Oberösterreich und Niederösterreich bereits als „Oberdonau“ und „Niederdonau“ bezeichnet wurden. Das Burgenland erschien als Teil Niederösterreichs, Vorarlberg als Teil Tirols. Die Karte „Deutsches Reich“ (S. 44) inkludierte neben Österreich das besetzte Tschechien („Protektorat Böhmen und Mähren“), große 
Teile Polens (nach dem Hitler-Stalin-Pakt 1939) sowie Luxemburg und Elsass-Lothringen. In der 61. Auflage 1944 war die Bezeichnung „Österreich“ durch „Die Heimat“ ersetzt. Die frühere Karte „Die Ostmark“ (S. 114/115) hieß nach der seit 1942 gültigen Sprachregelung nun „Die Alpen- und Donaugaue“. Die Eroberungen NS-Deutschlands in Osteuropa seit 1941 zeigte die Karte „Großdeutschland“ auf S. 44, die nunmehr Polen, Teile der Westukraine und Slowenien als Reichsterritorium auswies (Kozenn-Atlas, Ausgaben von 1941 bzw. 1944).

Während der Kriegsjahre 1939 bis 1945 vertrieb der Verlag Ed. Hölzel gemeinsam mit den Verlagen Franz Deuticke und Jugend und Volk die deutschen Geographieserien „Heimat und Welt“ sowie die „E. von Seydlitz“sche Erdkunde“ in Österreich. Der damalige Herausgeber des „Seydlitz“, der auf eines der erfolgreichsten Geographielehrwerke des 19. Jahrhunderts zurückgeht, war ein gewisser Walther JANTZEN (1904-1962), der als Mitarbeiter des NS-,,Zentralinstituts für Erziehung und Unterricht“ und „Schriftleiter“ der NS-Zeitschrift „Weltanschauung und Schule“ als überzeugter Nationalsozialist gelten kann (BIRSAK und WIESINGER 2019, S. 55).

Die Haupttätigkeit des Verlages zwischen 1939 und 1945 bestand allerdings in kartographischen Arbeiten für die Deutsche Wehrmacht. Zur Orientierung, insbesondere im osteuropäischen Kriegsgebiet, benötigten die NS-Truppen Karten. Spezielle „Kartentruppen im Osten“ hatten deshalb die Aufgabe, sowjetische Kartenbestände zu erbeuten. Diese wurden von kartographischen Verlagen in Deutschland und Österreich kopiert und - mit deutschen Erläuterungen versehen - nachgedruckt. $\left.{ }^{6}\right)$ Auf dieser Grundlage arbeiteten mehrere kartographische Verlage während der Kriegsjahre auch an einer Karte der Sowjetunion („Rußland“) im großen Maßstab 1 : 100 000. Außerdem arbeitete der Verlag Ed. Hölzel im Auftrag der Wehrmacht an einer militärgeographischen Karte des Donaudeltas, die allerdings nicht fertiggestellt wurde (BIRSAK und WIESINGER 2019, S. 54-55).

\section{Kartographische Produkte und Schulbücher seit 1945}

Im Jahr 1945 markierte das von dem späteren Univ.-Prof. Dr. Moshe BrawER initiierte Projekt „Israelischer Atlas“ den Neubeginn des Verlags Ed. Hölzel nach dem Zweiten Weltkrieg. Der 1919 in Wien geborene BraWER, Sohn des Geographen und Mitglieds der Geographischen Gesellschaft in Wien [ab 1959 Österreichische Geographische Gesellschaft) Dr. Abraham Brawer (1884-1975), kam 1945 als britischer Kriegskorrespondent nach Wien. Dem damaligen Geographiestudenten war der „Kozenn-Atlas“ wohlbekannt, und so kamen BRAwER und EckeLt 1945/46 überein, einen Atlas in hebräischer Sprache (Iwrit) für Schulen in Palästina zu produzieren. Seither stellte Ed. Hölzel mit BRAWER und einem israelischen Partnerverlag verschiedene sehr erfolgreiche Atlanten für israelische Schulen und Universitäten in hebräischer und arabischer Sprache sowie Wandkarten für den Schulgebrauch her (Abb. 10, BRAwER 2019).

Das bereits in der Zwischenkriegszeit erprobte und auch bei den israelischen Atlanten angewandte Geschäftsmodell, fremdsprachige Atlanten in Kooperation mit einheimischen Experten und Verlagen zu produzieren, wurde in den folgenden Jahrzehnten erfolgreich fortgeführt. Atlanten mit Karten aus dem Verlag Ed. Hölzel erschienen seit den 1950er Jahren in Deutschland, Belgien, Großbritannien, Frankreich, den Niederlanden, der Türkei (seit der Zwischenkriegszeit), den USA und Italien (Südtirol) sowie in Bulgarien (ab 2010). Eine besondere Kooperation war die Erarbeitung des wissenschaftlichen Atlas „Resources and Environment“ (1998) mit der Russischen Akademie der Wissenschaften (BIRSAK und WiESINGER 2019, S. 59ff, S. 77).

\footnotetext{
6) Im Verlagsarchiv finden sich neben zahlreichen kartographisch relevanten Broschüren von NS-Behörden auch sowjetische „Beutekarten“ und davon abgeleitete Karten.
} 


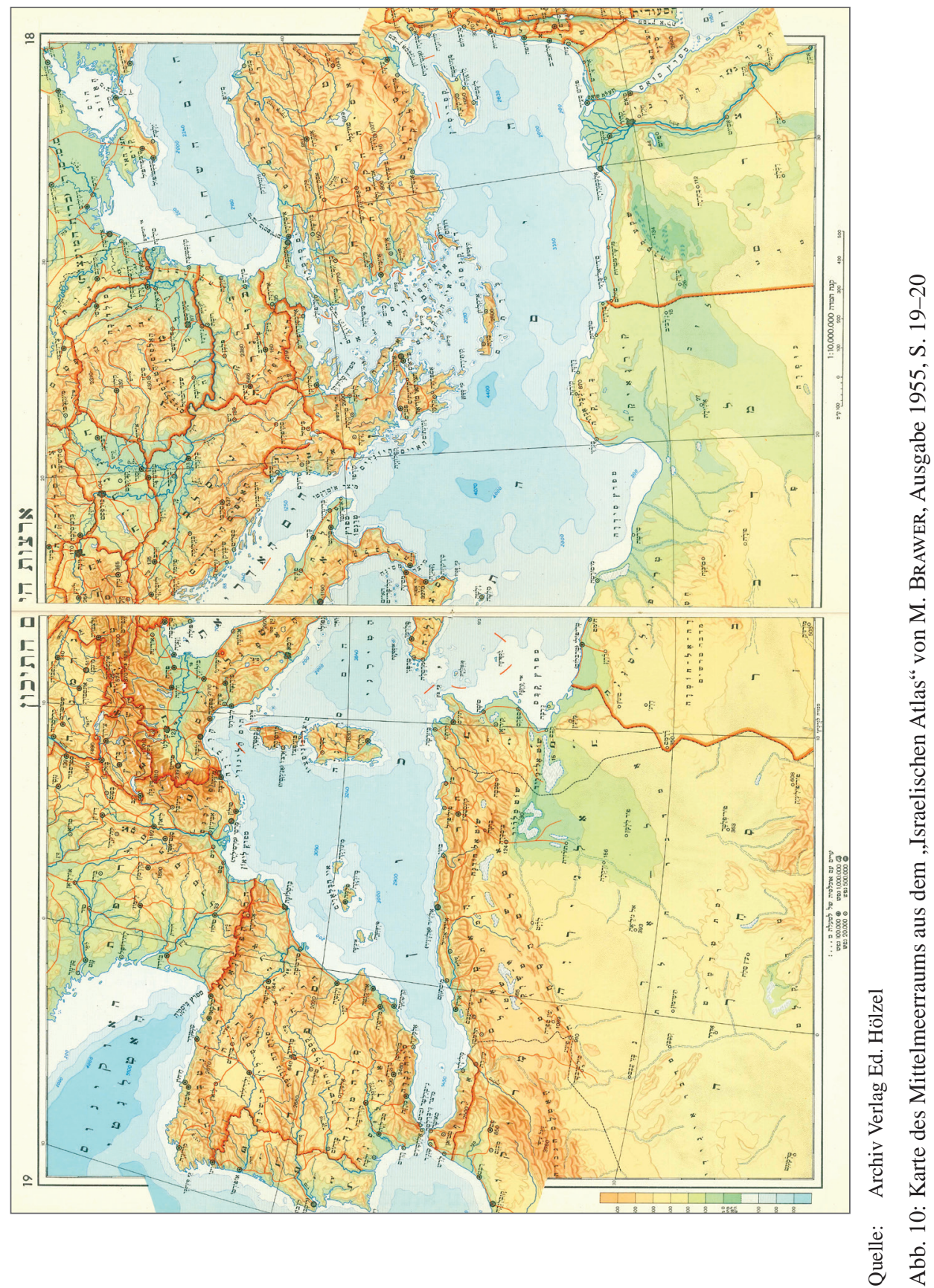


Angesichts der steigenden Motorisierung und zunehmenden Reiselust der Menschen in Europa nahm seit den späten 1950er Jahren die Bedeutung von Straßenkarten und Stadtplänen im Verlagssortiment zu. Gemeinsam mit dem französischen Partner Bordas erschienen unter dem Markennamen „Les Cartes Bleues“ seit 1958 Straßenkarten für West- und Südeuropa im Maßstab 1 : 500 000. In Österreich war der ÖAMTC der bedeutendste Kooperationspartner, für den Autokarten und insbesondere der „ÖAMTC Städteatlas Österreich“ (Erstauflage 1987) hergestellt wurden. Insbesondere von der „Straßenkarte Österreich 1 : 150 000“ wurden in den 1990er Jahren zahlreiche Nebenprodukte abgeleitet, etwa „Der große Straßenatlas Österreich“ und verschiedene Bundesländerausgaben. Seit dem Jahr 2000 wurden Straßenkarten aus dem Verlag Ed. Hölzel auch unter dem Markennamen „Euromaps“ vertrieben (BIRSAK und WIESINGER 2019, S. 61-81).

Teils gegenläufige Entwicklungen prägten die Verlagstätigkeit von Ed. Hölzel im Schulbuchbereich. Zwischen 1945 und 2005 gehörte der Verlag dem „Arbeitskreis österreichischer Schulbuchverleger“ an, der als genehmigtes Kartell den Schulbuchmarkt bestimmte. Ed. Hölzel bearbeitete in diesem Kontext die Fächer „Geographie und Wirtschaftskunde“ sowie in geringerem Ausmaß „Geschichte und Sozialkunde“. Der Wirtschaftsaufschwung der 1950er Jahre verstärkte die Nachfrage nach Schulbüchern, denn zunehmend konnten Eltern wieder in die Bildung ihrer Kinder investieren und ihnen neue Schulbücher kaufen.

Die wesentlichste Veränderung war sicherlich die Einführung der Österreichischen Schulbuchaktion 1972, die allen Schülerinnen und Schülern in Österreich die benötigten Schulbücher kostenlos zur Verfügung stellt. Durch die Schulbuchaktion und die allgemeine Bildungsexpansion seit den 1970er Jahren stieg die Nachfrage nach Schulbüchern wesentlich (BIRSAK und WIESINGER 2019, S. $57,59,67)$.

Nach der Auflösung des „Arbeitskreises“ 2005 entstand eine neue Konkurrenzsituation. Einerseits begann bzw. beschleunigte sich dadurch die Übernahme österreichischer Schulbuchverlage durch deutsche Konzerne. Andererseits differenzierte sich das Schulbuchangebot dadurch wesentlich aus. Derzeit stehen etwa für den Unterricht in „Geographie und Wirtschaftskunde“ in der Unterstufe 18 Serien und 12 Atlanten (auch fächerübergreifend) zur Auswahl, in der Oberstufe (nur allgemeinbildende höhere Schule, AHS) sind es immerhin sechs Serien und sechs Atlanten (auch fächerübergreifend) (Schulbuchlisten 2019/20). Die durch dieses Überangebot reduzierten Absatzchancen beeinflussen natürlich die Konzeption neuer Produkte durch die Verlage erheblich.

Für die Hölzel'schen Schulatlanten war zwischen 1949 und 1984 Hans SLANAR jun. (19241999) als wissenschaftlicher Leiter der Kartographie hauptverantwortlich. Die erste Nachkriegsausgabe des bisherigen „Kozenn-Atlas“ erschien in der Bearbeitung von Dr. Hans SlanaR sen. 1951 unter dem neuen Titel „Österreichischer Mittelschulatlas“. SLANAR sen. leitete davon auch den „Österreichischen Hauptschulatlas“ ab, der zeitgleich in fünf Bundesländerausgaben erschien, und verfasste „Methodische Ratschläge“ (1952) für die praktische Atlasarbeit in beiden Schultypen. In den 1960er Jahren übernahmen Univ.-Prof. Dr. Walter STRZYGowski (1908-1970) und Univ.-Prof. Dr. Wigand RitTER (1933-2013) von der Hochschule für Welthandel bzw. Wirtschaftsuniversität Wien die Bearbeitung des „Mittelschulatlas“, wobei Wirtschaftskarten immer wichtiger wurden (BIRSAK und Wiesinger 2019, S. 59, 63).

Im Jahr 1968 erfolgte eine erneute Umbenennung in „Österreichischer Atlas für höhere Schulen“. Dieser Namensänderung lag die Reform des österreichischen Schulwesens durch das Schulorganisationsgesetz 1962 zugrunde, demzufolge die bisherigen „Mittelschulen“ in „Allgemeinbildende höhere Schulen“ umbenannt wurden (LoEw o. J.). Der „Österreichische Hauptschulatlas“ wurde für die Ausgabe 1972 von SLANAR jun. et al. aktualisiert und erweitert.

1978 erstellte das Unterrichtsministerium erstmals einen verbindlichen Themenkatalog für Schulatlanten und schrieb anstelle der Differenzierung nach Schultypen die Differenzierung nach Schulstufen vor. Dementsprechend hieß der ehemalige „Hauptschulatlas“ seit der Neubearbeitung 
1979 „Österreichischer Unterstufenatlas“. Dieser war übrigens der erste österreichische Schulatlas mit einem Namenregister (SitTe W. 2001, S. 417). 1981 folgte dann der „Österreichische Oberstufenatlas“, konzipiert von SLANAR jun., den neue Wirtschaftskarten von RitTER ergänzten (BIRSAK und Wiesinger 2019, S. 71; SitTe W. 2001, S. 418).

1985 wurden die Lehrpläne für „Geographie und Wirtschaftskunde“ in der Unterstufe grundlegend erneuert. Die 1989 erschienene Neubearbeitung des „Österreichischen Unterstufenatlas“ durch Dr. Lukas BIRSAK berücksichtigte bereits diese neuen Lehrpläne. In den 1990er Jahren entstanden zahlreiche neue Hölzel-Atlanten unter der Leitung von BIRSAK. Den Anfang machten 1995 der Atlas „Hölzel 5/8“ (für die 5. bis 8. Schulstufe, also Unterstufe) und der „Hölzel-Weltatlas“ für die Oberstufe. Der „Hölzel 5/8“ von BIRSAK et al. zeichnete sich durch den neuartigen Einsatz von Abbildungen auf bzw. zu den Karten aus, die die Atlasarbeit anschaulicher machen sollten (Abb. 11).

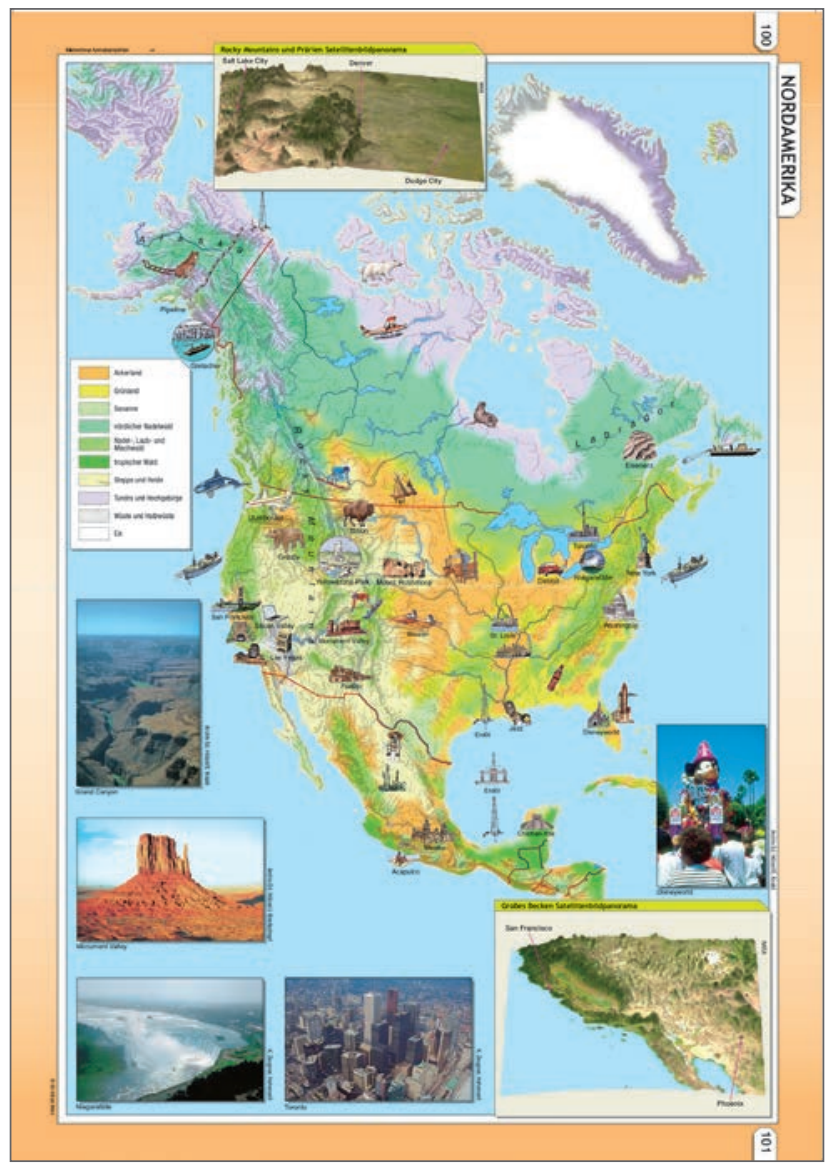

Die Karte wirkt durch Illustrationen und Fotografien anschaulicher.

Quelle: Archiv Verlag Ed. Hölzel

Abb. 11: „Bilderkarte Nordamerika“ aus dem Atlas „Hölzel 5/8“, Ausgabe 2019, S. 100-101 
Im Jahr 1996 kam erstmals seit den 1970er Jahren wieder ein „Neuer Kozenn-Atlas“ heraus. 2004 erschien unter dem Titel „Hölzel Universalatlas zu Geographie und Geschichte“ der erste fächerübergreifende Atlas bei Ed. Hölzel, der für zahlreiche Schultypen (Unter- und Oberstufe) approbiert wurde. Bis 2008 wurde mit dem „Hölzel-Kombiatlas“ ein Geschichte- und Geographieatlas speziell für die Unterstufe entwickelt. Zum 150-jährigen Jubiläum des „Kozenn-Atlas“ entstand 2011 der „Große Kozenn-Atlas“, der einen extra gebundenen Geschichteteil und eine Atlas-CD enthielt. Dieses Werk wurde auf der 25. Konferenz der International Cartographic Association mit dem 1. Preis für gedruckte Atlanten ausgezeichnet (BIRSAK und WIESINGER 2019, S. 77, 83, 85; allgemeiner auch BirSAK 1996 und KNABL 2011).

Die erste neue Lehrbuchserie nach 1945 war die von Hans Fuchs et al. verfasste „Erdkunde“ für Haupt- und Mittelschulen (i. e. Gymnasien) in acht Teilen, die zwischen 1946 und 1953 in Verlagsgemeinschaft mit den Verlagen Jugend und Volk, Deuticke und Österreichischer Bundesverlag (ÖBV) bei Ed. Hölzel erschien. Auf einen mit nur wenigen Illustrationen aufgelockerten Textteil folgt in dieser Serie stets ein Abbildungsteil mit zahlreichen Schwarz-Weiß-Fotografien. Konzeptionell und inhaltlich geht die „Erdkunde“ auf das ebenfalls mehrteilige „Arbeits- und Lernbuch der Erdkunde“ von Fuchs und SLANAR sen. aus der Zwischenkriegszeit zurück (Fuchs et al. 1946 etc.).

Ende der 1950er Jahre erschien in Verlagsgemeinschaft mit Franz Deuticke, ÖBV (der ab den 1970er Jahren nicht mehr mitwirkte) und Jugend und Volk nach dem unrühmlichen Vorspiel in der NS-Zeit eine neue österreichische Lizenzausgabe der deutschen Geographie-Serie „Seydlitz“ für die Unter- und Oberstufe. Dieses Lehrbuch wurde bis in die 1980er Jahre aufgelegt. Herausgeber war bis in die 1970er Jahre Univ.-Prof. Dr. Leopold ScHEIDL (1904-1974) von der Hochschule für Welthandel, der mit einem Team von Autoren zusammenarbeitete. Im „Seydlitz“ der 1950er Jahre dominierten ausführliche, aber gut verständliche Informationstexte, die von Aufgaben zur Orientierung im Raum (Rubrik „Ein Blick auf die Karte“) eingeleitet und von wenigen Arbeitsaufgaben abgeschlossen wurden. Ein positives Merkmal waren sicherlich die zahlreichen Abbildungen (hauptsächlich Schwarz-Weiß-Fotos) im Text, welche die dichten Informationen auflockerten und veranschaulichten (SCHEIDL et al. 1959).

Auch im „Seydlitz“ der 1970er Jahre überwog noch der Informationstext. Dieser war allerdings durch Überschriften und Zwischenüberschriften sowie fett oder gesperrt hervorgehobene Schlüsselbegriffe gut gegliedert. Farbige Illustrationen und Fotografien - zu dieser Zeit eine Besonderheit - veranschaulichten die Textinhalte und sorgten für einen angenehmeren Gesamteindruck des Werks. Blau hinterlegte Kästen mit Zusammenfassungen sollten den Schülerinnen und Schülern das Lernen erleichtern. Die Arbeitsaufgaben waren übersichtlich am Ende jedes Unterkapitels zusammengefasst. Schon die dafür gewählte kleinere Schrift wies allerdings auf ihre im Vergleich zum Informationstext untergeordnete Bedeutung im Lehrwerkskonzept (und wohl ebenso im damaligen Unterricht) hin. Reproduzierende Aufgaben, die Sachwissen abfragten, standen im Vordergrund; anspruchsvollere Aufgaben, die etwa Vergleiche, Begründungen und die Bildung von Hypothesen anregten, kamen seltener vor. Ein kurios-sympathisches Detail: Die Unterkapitel im Kapitel „Unsere Republik“ trugen als Überschriften Verse aus der Bundeshymne: „Land der Berge“, „Land am Strome“ usw. (ScHEIDL et al. 1973).

Wesentliche Neuerungen bei den Geographieschulbüchern brachten erst die 1980er Jahre. Schon vor Einführung der neuen Lehrpläne für „Geographie und Wirtschaftskunde“ hatte der Verlag Ed. Hölzel in Zusammenarbeit mit Versuchsschulen neue Lehrwerke entwickelt, die dann 1985 mit Inkrafttreten der neuen Lehrpläne in Verlagsgemeinschaft mit Franz Deuticke, Leykam und Jugend und Volk erschienen: die Schulbuchreihen „Standpunkte“ für die AHS-Unterstufe und „Lebensräume“ für die Hauptschulen, beide von Harald Hitz et al. Diese Serien waren nach Themen strukturiert, aufgabenzentriert und schülerorientiert. Dadurch unterschieden sie sich wesentlich von den 
bis dahin üblichen, länderkundlich aufgebauten und auf den Informationstext fokussierten Geographieschulbüchern. Mit zahlreichen Grafiken, Cartoons und Abbildungen wollten „Standpunkte“ und „Lebensräume“ die Schülerinnen und Schüler auch optisch ansprechen. Durch die Serien führten verschiedene Leitcharaktere (der Außerirdische Lo, der Pirat Säbel-Otto, realistische Jugendliche ...), die die Welt erforschten, beschrieben und erklärten.

Die vielfältigen Textsorten im Schulbuch (fiktive Erzählungen, Berichte, Interviews usw.) rekurrierten inhaltlich und sprachlich auf die Lebenswelt der Schülerinnen und Schüler und sorgten für Abwechslung. Die meisten Texte waren anschaulich illustriert. Sie vermittelten damit Informationen auf zielgruppengerechte, oft auch unterhaltsame Weise. Der rein sachlich-informierende Autorentext konnte so knapper gehalten werden. Er war in kurze, nummerierte Abschnitte gegliedert und wurde durch Arbeitsaufgaben sowohl aufgelockert als auch inhaltlich vertieft. Bedingt durch die Vielzahl der Leitcharaktere, Abbildungen und Textkategorien geriet das Layout jedoch etwas unübersichtlich, sodass fraglich scheint, wie gut sich die Nutzer der Bücher darin zurechtfanden.

Die eher schmalen Lehrbücher der Serien „Standpunkte“ und „Lebensräume“ wurden durch zahlreiche Arbeitsblätter ergänzt. Ein umfangreicher Lehrerband sollte den Lehrerinnen und Lehrern die Umstellung auf das neue Unterrichts- und Lehrwerkskonzept mit den Schwerpunkten Themen-, Aufgaben- und Schülerzentrierung erleichtern (Hitz et al. 1985a, 1985b, 1986a, 1986b, 1987).

Um das Jahr 2000 erschienen mit „Hölzel-GW 1/Faszination Erde“ von K. ZeuGNER und „panorama.at" von einem Autorenteam um L. BAUER wieder neue Lehrwerke, wobei letzteres als erste Hölzel-Schulbuchserie das Internet für zusätzliche Aufgaben und Spielideen zum Buch nutzte (BIRSAK und Wiesinger 2019, S. 77, 81; ZeUGNER 1998; BAUER et al. 2002).

Seither differenzierte sich das Schulbuch-Angebot des Verlages Ed. Hölzel weiter aus und umfasst derzeit acht Geographie-Serien für die Unterstufe, vier Geographie-Serien für die AHS-Oberstufe und neun Serien für die berufsbildenden mittleren und höheren Schulen (Verlag Ed. Hölzel 2019).

\section{Digitale Bildungsmedien aus dem Verlag Ed. Hölzel}

In den 1990er Jahren erfolgte die Umstellung auf digitale Produktionsmethoden in Kartographie und Satz sowie die Konzeption und Produktion erster digitaler Bildungsmedien im Verlag Ed. Hölzel (KNABL 1999). Der „GEOTHEK Weltatlas“ auf CD-ROM war der erste deutschsprachige digitale Weltatlas, der zu den Karten verknüpfte Fotos, Diagramme und Statistiken enthielt. Auf diesen Atlas geht das heutige umfangreiche digitale Angebot zu den Hölzel-Atlanten zurück. In Verlagsgemeinschaft mit dem Österreichischen Bundesverlag und Hölder, Pichler, Tempsky (öbv \& hpt) erschien 2000 die CD-ROM „KLIO - multimediale Weltgeschichte mit dynamischem Atlas“.

Seither wurden CD-ROMs zunehmend durch internetbasierte Anwendungen abgelöst. 2002 bot die Unterstufen-Reihe „panorama.at“ von L. BAUER et al. als eine der ersten in Österreich interaktive Lernspiele auf einer eigenen Homepage zum Buch an. Seit 2015 unterstützt Ed. Hölzel unter dem Markennamen „digiHölzel“ Lehrkräfte mit E-Books, die vielfältige Zusatzmaterialien für den Unterricht enthalten. Seit 2016 sind E-Books unter der Bezeichnung „DIGI4SCHOOL“ auch für Schülerinnen und Schüler über die Schulbuchaktion erhältlich (BIRSAK und WIESINGER 2019, S. 75, 81, 85, 87). Dasselbe gilt auch für Schulatlanten aus dem Verlag Ed. Hölzel. So enthält beispielsweise das „digiHölzel“-E-Book zum „Hölzel-Aktivatlas“ von Ludwig BiRsAK und Johannes MAYER für die Unterstufe Arbeitsblätter, Kopiervorlagen und Lösungen für die Lehrkräfte; Schülerinnen und Schüler erhalten das E-Book zum Atlas kostenlos. Der Atlas „Hölzel 5/8“ für die Unterstufe wiederum liegt nicht nur als erweitertes E-Book für Lehrkräfte und einfaches E-Book für Schülerinnen und Schüler vor, sondern ist wahlweise auch mit der CD „Runde Sache - Faszina- 
tion Erde“ von K. Zeugner bestellbar, die Rätsel, Aufgaben und Spiele zur Atlasarbeit enthält. Der schulstufen- und schultypenübergreifende „Hölzel-Universalatlas zu Geographie und Geschichte“ schließlich bietet zusätzlich zu „digiHölzel“ und „DIGI4SCHOOL“ noch die „GEOTHEK Schulatlas-CD“, die praktisches Arbeiten mit digitalen Karten und aktuellen Geodaten ermöglicht. Diese Beispiele sind nur ein kleiner Ausschnitt aus dem digitalen Medienangebot von Ed. Hölzel, das kontinuierlich weiterentwickelt wird.

\section{Resümee}

Der 1844 gegründete Verlag Ed. Hölzel war bereits im 19. Jahrhundert ein führender österreichischer Verlag für (Schul-)Geographie und (Schul-)Kartographie. Historische und geographische Wandbilder, Wandkarten, Schulatlanten (für Geographie und Geschichte) sowie Schulbücher bilden seither einen Schwerpunkt der Verlagstätigkeit, die außerdem auch Autokarten, wissenschaftliche Atlanten, Fach- und Reiseliteratur sowie Kalender umfasst. Heute bietet Ed. Hölzel Schulbücher für unterschiedlichste Fächer und Schultypen an und entwickelt gemeinsam mit dem früheren MANZ Verlag Schulbuch als Schwester digitale Bildungsmedien für das 21. Jahrhundert.

\section{Literaturverzeichnis}

Althaus Th. (2018): Darstellungsoptik, Bild-Erfassung und Bilderfülle in der Prosa des 19. Jahrhunderts. Bielefeld: Aisthesis.

BAuER L. et al. (2002): panorama.at 1. Wien: Ed. Hölzel/Leykam/ÖBV \& hpt/Jugend \& Volk.

BIRSAK L. (1996): Der Verlag Ed. Hölzel - 150 Jahre Kartographie aus Österreich. In: KRETSCHMER I, KRIZ K. (Hrsg.): Kartographie in Österreich '96. Wien: Geographisches Institut der Universität Wien, S. 165-173 (= Wiener Schriften zur Geographie und Kartographie, 9).

BIRSAK L. (2011): Kozenn-Atlas. Eine kurze Geschichte zum 150. Geburtstag eines Meilensteins der österreichischen Schulkartographie. Wien: Ed. Hölzel.

BirsaK L., WiesINGer B. N. (2019): Chronologischer Abriss der Verlagsgeschichte. In: Innovation aus Tradition. Festschrift 175 Jahre Ed. Hölzel. Wien: Ed. Hölzel, S. 8-87.

Bratec Mrvar R. et al. (2011): Kocenov srednješolski atlas kot didaktična prelomnica [Kozenns Mittelschulatlas als didaktischer Wendepunkt]. Ljubljana: Založba ZRC.

Brawer M. (2019): Der „Israelische Atlas“: Die Geschichte eines Atlas seit 1945. In: Innovation aus Tradition. Festschrift 175 Jahre Ed. Hölzel. Wien: Ed. Hölzel, S. 106-111.

Dammerer F. P. (2007): Vinzenz von Haardt (1843-1914). Ein Kartograph der Franzisko-Josephinischen Zeit, der „die vollendete Technik der Kartographie mit pädagogischem Geschicke vereint“. Dissertation, Universität Wien.

Fuchs H. et al. (1946 etc.): Erdkunde. Wien: F. Deuticke/Ed. Hölzel/ÖBV/Jugend und Volk.

HeIDERICh F. ( $\left.{ }^{4} 1910\right)$ : Österreichische Schulgeographie. Erster Teil für die I. Klasse der Mittelschulen. Wien: Ed. Hölzel.

Hitz H. et al. (1985a): Lebensräume 1. Wien: F. Deuticke/Ed. Hölzel/Jugend und Volk/Leykam.

Hitz H. et al. (1985b): Standpunkte 1. Wien: F. Deuticke/Ed. Hölzel/Jugend und Volk/Leykam.

Hitz H. et al. (1986a): Lebensräume 2. Wien: F. Deuticke/Ed. Hölzel/Jugend und Volk/Leykam.

Hitz H. et al. (1986b): Lebensräume 2 Arbeitsblätter. Wien: F. Deuticke/Ed. Hölzel/Jugend und Volk/Leykam.

Hitz H. et al. (1987): Lebensräume 2 Lehrerband. Wien: F. Deuticke/Ed. Hölzel/Jugend und Volk/ Leykam. 
Hroch M. ('1996): Sprache, Literatur und nationale Identität. In: Nautz N., VahrenKamp R. (Hrsg.): Die Wiener Jahrhundertwende. Einflüsse, Umwelt, Wirkungen. Wien/Köln/Graz: Böhlau, S. 377-388.

JovANOVIC-KRUSPEL S. (2019): Bilder der Welt: Hölzels „Geographische Charakterbilder“ zwischen Museum, Schule und Wohnzimmer. In: Innovation aus Tradition. Festschrift 175 Jahre Ed. Hölzel. Wien: Ed. Hölzel, S. 99-105.

KLEE A. (2019): Die Verlegerfamilie Hölzel: Ein Netzwerk der Kultur. In: Innovation aus Tradition. Festschrift 175 Jahre Ed. Hölzel. Wien: Ed. Hölzel, S. 96-98.

KnABL E. (1999): Vom Gravurring zur Maus - Kartentechnik im Umbruch. In: Kretschmer I., KrIz K. (Hrsg.): 25 Jahre Studienzweig Kartographie. Wien: Geographisches Institut der Universität Wien, S. 136-148 (= Wiener Schriften zur Geographie und Kartographie, 12).

Knabl E. (2011): Kartographie aus dem Verlag Ed. Hölzel. In: Kainz W., Kriz K., Riedl A. (Hrsg.): 50 Jahre Österreichische Kartographische Kommission. Jubiläumsband zum Festsymposium, 10.-11. November 2011. Wien: Institut für Geographie und Regionalforschung der Universität Wien, S. 1-12 (= Wiener Schriften zur Geographie und Kartographie, 20).

KubičKovÁ P. (2017a): Zum 200. Geburtstag des Verlegers Eduard Hölzel und zu seinen Anfängen in Olmütz. In: Mitteilungen der Österreichischen Geographischen Gesellschaft, 159, S. 348-358.

KuBIČKová P. (2017b): Olomoucky nakladatel Eduard Hölzel [Der Olmützer Verleger Eduard Hölzel]. In: KubičKová P., Gionek J., Krušinský R., NovotnÝ L.: Eduard Hölzel 1817-1885. Katalog výstavy 2.11.2017-29.12.2017 [Katalog zur Ausstellung 2.11.2017-29.12.2017]. Olomouc [Olmütz]: Vědecká knihovna v Olomouci [Forschungsbibliothek Olmütz], S. 9-31.

KuBIČKovÁ P. (2017c), Odborné publikace, beletrie, litografie [Ausgewählte Veröffentlichungen, Belletristik, Lithographien]. In: KubičKová P., GioneK J., KrušInskÝ R., Novotný L.: Eduard Hölzel 1817-1885. Katalog výstavy 2.11.2017-29.12.2017 [Katalog zur Ausstellung 2.11.2017-29.12.2017]. Olomouc [Olmütz]: Vědecká knihovna v Olomouci [Forschungsbibliothek Olmütz], S. 60-116.

KuBIČKová P. (2019): Eduard Hölzel: Ein erfolgreicher Verleger zwischen Olmütz und Wien. In: Innovation aus Tradition. Festschrift 175 Jahre Ed. Hölzel. Wien: Ed. Hölzel, S. 92-95.

LoEw M. (о. J.): Das Schulorganisationsgesetz 1962. - https://austria-forum.org/af/Wissenssamm lungen/Essays/Bildung/Das_Schulorganisationsgesetz_1962 (Zugriff: 15.10.2019).

ScheIdL L. et al. (1959): Seydlitz - Lehrbuch der Erdkunde. 2. Teil für die zweite Klasse der Hauptund Mittelschulen. Wien: F. Deuticke/Ed. Hölzel/ÖBV/Jugend und Volk.

ScheIDL L. et. al. (1973): Seydlitz - Lehrbuch der Geographie und Wirtschaftskunde. 1. Teil für die erste Klasse der Hauptschulen und AHS. Wien: Ed. Hölzel/F. Deuticke/Jugend und Volk.

Schulbuchliste 2019/20 (Sek. II). - https://www.schulbuchaktion.at/sba_downloads/sba2019/ 1000_1100_sbl2019.pdf (Zugriff: 18.10.2019).

Schulbuchliste 2019/20 (Sek. I). - https://www.schulbuchaktion.at/sba_downloads/sba2019/0300_ sbl2019.pdf (Zugriff: 18.10.2019).

SitTe Ch. (1987): Bibliographie zur Entwicklung der Schulbücher aus Geographie (Geographie und Wirtschaftskunde) in Österreich. In: Mitteilungen der Österreichischen Geographischen Gesellschaft, 129, S. 128-165.

Sitte W. (2001): Schulatlas I (Zu seiner Geschichte in Österreich). In: Sitte W., Wohlschlägl H. (Hrsg.): Beiträge zu Didaktik des „Geographie und Wirtschaftskunde”-Unterrichts. Wien: Institut für Geographie und Regionalforschung der Universität Wien, S. 410-423 (= Materialien zur Didaktik der Geographie und Wirtschaftskunde, 16). 
Verlag Ed. Hölzel (1906): Verlags-Katalog. Wien: Ed. Hölzel.

Verlag Ed. Hölzel (2019): Verlagskatalog 2019/20. Wien: Ed. Hölzel.

Vuković M. (2019): Von wunderbarer Klarheit. Friedrich Simonys Gletscherfotografien 18751891. Wien: Photoinstitut Bonartes/Album Verlag.

Zeugner K. (1998): Hölzel-GW 1. Faszination Erde. Wien: Ed. Hölzel/ÖBV \& hpt/Leykam. 